Article

\title{
Optimal Fluorescence Waveband Determination for Detecting Defective Cherry Tomatoes Using a Fluorescence Excitation-Emission Matrix
}

\author{
In-Suck Baek ${ }^{1}$, Moon S. Kim ${ }^{2}$, Hoosoo Lee ${ }^{1}$, Wang-Hee Lee ${ }^{1}$ and Byoung-Kwan Cho ${ }^{1, *}$ \\ 1 Department of Biosystems Machinery Engineering, College of Agricultural and Life Science, \\ Chungnam National University, 99 Daehak-ro, Yuseong-gu, Daejeon 305-764, Korea; \\ E-Mails: huntersp@naver.com (I.-S.B.); benet33@naver.com (H.L); wanghee@cnu.ac.kr (W.-H.L.) \\ 2 Environmental Microbiology and Food Safety Laboratory, Agricultural Research Service, U.S. \\ Department of Agriculture, Powder Mill Road, Building \#303, BARC-East, Beltsville, MD 20705, \\ USA; E-Mail: moon.kim@ars.usda.gov
}

* Author to whom correspondence should be addressed; E-Mail: chobk@ cnu.ac.kr; Tel.: +82-42-821-6715; Fax: +82-42-823-6246.

External Editor: Gonzalo Pajares Martinsanz

Received: 8 August 2014; in revised form: 15 October 2014 / Accepted: 30 October 2014 /

Published: 14 November 2014

\begin{abstract}
A multi-spectral fluorescence imaging technique was used to detect defective cherry tomatoes. The fluorescence excitation and emission matrix was used to measure for defects, sound surface and stem areas to determine the optimal fluorescence excitation and emission wavelengths for discrimination. Two-way ANOVA revealed the optimal excitation wavelength for detecting defect areas was $410 \mathrm{~nm}$. Principal component analysis (PCA) was applied to the fluorescence emission spectra of all regions at $410 \mathrm{~nm}$ excitation to determine the emission wavelengths for defect detection. The major emission wavelengths were $688 \mathrm{~nm}$ and $506 \mathrm{~nm}$ for the detection. Fluorescence images combined with the determined emission wavebands demonstrated the feasibility of detecting defective cherry tomatoes with $>98 \%$ accuracy. Multi-spectral fluorescence imaging has potential utility in non-destructive quality sorting of cherry tomatoes.
\end{abstract}

Keywords: cherry tomato; quality sorting; defect detection; hyperspectral image; fluorescence image 


\section{Introduction}

International agricultural quality competitions have become a popular way to increase free trade between nations. Consumption and exports of cherry tomatoes are increasing as consumers become more health-conscious and seek convenient healthy foods. Cherry tomatoes produced in Korea are largely exported to other Asian countries. Quality and safety are important concerns for produce exports. Selecting quality produce and careful shipping are critical to maintaining a competitive edge in export countries. There are far fewer methods for quality selection in cherry tomatoes than there are in standard tomatoes.

Cherry tomato quality is measured by peel coloration, shape, size, cracking, and sugar/acid content; however, current selection techniques typically involve simple size and shape discrimination with a drum selector. There is no more comprehensive technology for measuring quality in this product. Cracked tomatoes swiftly reduce quality when they are not detected prior to distribution, because infections lodged in the cracks are spread throughout the batch, reducing unit sales. Thus, cracked tomatoes must be selected and removed prior to shipment. Selection of cherry tomatoes is problematic due to their small size and large quantity, which prohibits the use of selection technologies used in standard tomatoes. Current nondestructive selection technologies employ spectroscopy and mechanical visualization, although these methods are difficult to apply at the site of production. Spectroscopy requires frequent recalibration and repeated sample measurements, and only measures a fixed point, rather than the entire surface of the fruit [1]. Mechanical visualization can be used to observe the entire fruit surface, but can provide only basic surface information. In cracked tomatoes, the surface and interior colors are similar, making general color imaging difficult. Thus, a new nondestructive selection technology is needed.

Hyperspectral image is a novel technology that collects image data at a series of narrow and contiguous wavelength bands for each spatial pixel of a captured scene, constructing 3-dimensional hyperspectral cube, to acquire both spatial and spectral information simultaneously [2]. As the spectrum of each spatial pixel can characterize the target substances at corresponding spot on the hyperspectral image, this technology can be used to identify and detect spectral and spatial anomalies in agricultural products [2]. Hyperspectral imaging can provide physical and chemical information beyond that provided by the simple optical R/G/B regions used by standard color cameras. It is even possible for the hyperspectral fluorescence characteristics of an object to yield a detailed image [3]. Hyperspectral fluorescence imaging technology combines fluorescence and digital imaging to provide simultaneous physical and chemical data. The technology is used for various applications, including diagnostics, remote imaging, and agricultural quality and safety monitoring. Agricultural applications of multispectral imaging include detection of apples infected with stock stool [4], detection of internal organ residue on the surface of chicken carcasses [5,6], and assessment of pickle quality [7].

The light source is very important in hyperspectral fluorescence techniques, because powerful, narrowband excitation is required to yield sufficient light emission from the sample [8]. Emission intensity is greatly influenced by excitation power and exposure time. Thus, the optimal excitation wavelength and a powerful light source must be identified in the context of the fluorescence characteristics of the sample type.

We validated the optimal excitation and emission wavelengths for detection of cracked cherry tomatoes by fluorescence imaging. The specific objectives were: (1) to measure the fluorescence 
characteristics of cherry tomato (i.e., sound surface, stems, cracks) at excitation wavelengths between $200 \mathrm{~nm}$ and $675 \mathrm{~nm}$ and emission wavelengths between $225 \mathrm{~nm}$ and $700 \mathrm{~nm}$; (2) to use a hyperspectral fluorescence imaging system equipped with an optimal light source for detection; and (3) to establish appropriate image processing and detection algorithms for defective cherry tomatoes.

\section{Materials and Methods}

\subsection{Sample Preparation}

'Koko' cherry tomatoes were harvested from a farm in Buyeo, Chungnam, South Korea. Cherry tomatoes were placed in plastic bags with ice and transported to the laboratory. The harvests were divided into groups. The first group contained 16 naturally cracked cherry tomatoes by random sampling and was used to identify the fluorescence characteristics of each part of the tomato. The second group consisted of 121 cracked and 23 normal cherry tomatoes, used to validate the selection accuracy of multispectral fluorescence imaging. Cuticle defects were arranged in an upward-facing position for ideal imaging.

\subsection{Measurement of Fluorescence Spectra}

Normal cuticle and stem parts and released matrix, fluoroMate FS-2 (Scinco Co. Seoul, Korea) were used to measure cuticle defects. To measure the fluorescence characteristics of each part of the fruit, samples were thinly cut and measured after placing the samples in circular cells made of a non-fluorescent substance as in Figure 1. A fluorophotometer measures fluorescence by measuring emitted photons at the detection wavelength. In this study, the fluorometer photo multiplier tube (PMT) was 700 volts and integration time was $20 \mathrm{msec}$. Excitation wavelengths from $200 \mathrm{~nm}$ to $665 \mathrm{~nm}$ were emitted by $150 \mathrm{~W}$ continuous xenon lamp and $5 \mathrm{~nm}$ intervals; emitted light from the sample was measured in $1 \mathrm{~nm}$ intervals from $225 \mathrm{~nm}$ to $700 \mathrm{~nm}$. Relative Fluorescence Intensity (RFI) measured across these wavelengths was used to build an Excitation-Emission matrix (EEM).

\subsection{Measurement of Fluorescence Images}

The hyperspectral imaging system was used to acquire fluorescence images as shown in Figure 2. The system was designed for use at $450 \mathrm{~nm}$ to $700 \mathrm{~nm}$ [9]. The system includes an electron multiplying charge-coupled device camera (EMCCD: Luca R DL-604M, 14-bit, Andor Technology, South Windsor, CT, USA), a C-mount objective lens (F1.9 35-mm compact lens, Schneider Optics, Hauppauge, NY, USA), imaging spectrograph (VNIR, Headwall photonics, Fitchburg, MA, USA), programmable uniaxial stage, and light sources (Figure 2A). Emission intensity is determined by the power of the light source and exposure time; thus, a real-time detection system must have a proper light source and short exposure time. To obtain sufficient excitation for imaging, we used five $410 \mathrm{~nm} 10 \mathrm{~W}$ LEDs (LZ4-40UA10, Ledengin Inc., Mansfield, OH, USA). As in Figure 2A, a pair of light sources was used. Wavelengths over $430 \mathrm{~nm}$ and the long wavelength spectral tail from the LEDs were blocked by a low-pass filter ( $<445 \mathrm{~nm}, 70 \%$ transmission with $450 \mathrm{~nm}$ cut-off) in front of the LEDs. Only spectral signals up to $700 \mathrm{~nm}$ were acquired, eliminating second-order excitation effects. The dimension of the light source 
was $250 \mathrm{~mm}$ (Length) $\times 100 \mathrm{~mm}$ (Width) $\times 150 \mathrm{~mm}$ (Height). Selecting the wavelength of LED excitation was performed by fluorophotometry (details provided in the Results and Discussion section).

Figure 1. Photos of cell unit (A); precision cell with samples (B).

A

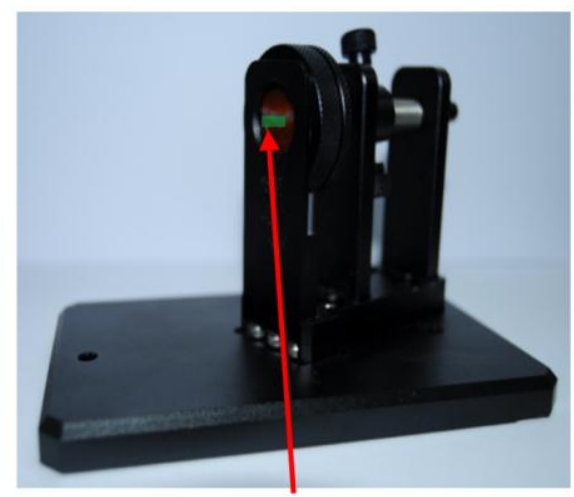

Excitation into focus

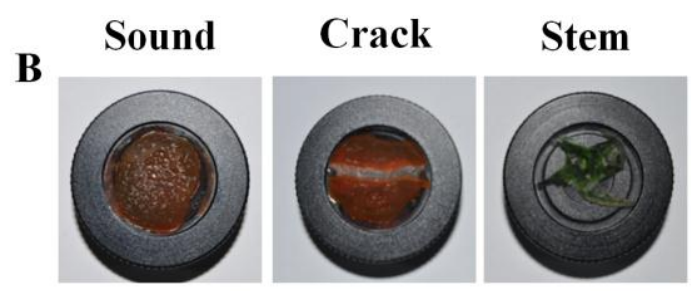

Visual Basic 6.0 was used to run the hyperspectral imaging system. To set the exposure time for hyperspectral imaging, the step motor was employed by automatically calculating the motion and exposure times. The image was projected through a C-mount objective lens and high-pass $460 \mathrm{~nm}$ filter (>455 nm, 70\% transmission with $460 \mathrm{~nm}$ cut-off), a slit, and imaging spectrograph. The instantaneous field of view (IFOV) of the system was limited to a thin line through the spectrograph aperture slit $(60 \mu \mathrm{m} \times 18 \mathrm{~mm})$ between the $\mathrm{C}$-mount objective lens. Imaging then comprised each spatial location on the IFOV of a line-scan and spectral information at each wavelength was determined by the spectrograph. The two-dimensional spectral and spatial data on the IFOV were captured by the EMCCD and stored as an image in the computer. The collected two-dimensional data was used to generate a 3D fluorescence hypercube (Figure 2B).

Spectral calibration was performed using a general-purpose cool-white fluorescent lamp with emitted wavelengths for terbium $\left(\mathrm{Tb}^{3+}\right)$, europium $\left(\mathrm{Eu}^{3+}\right)$, and mercury $(\mathrm{Hg})$. The white Teflon panel emitted a peak wavelength after illumination with the cool-white fluorescent lamp and numbers in the spectral dimension of the hyperspectral system corresponded to actual wavelength peaks from the cool-white fluorescence lamp after linear regression.

In this study, the exposure time was $0.1 \mathrm{~s}$ and the motor moved at $1 \mathrm{~mm}$ intervals for $250 \mathrm{steps}$. We obtained a full hyperspectral image of $25 \mathrm{~cm}$ (whole sample). Images provided mass information, known as a 3D fluorescence hypercube. The hypercube provides 2D fluorescence imaging (from $470 \mathrm{~nm}$ to $700 \mathrm{~nm}$ ) 
and spectral information (Figure 2B). The optimum wavelength range for defect tomato detection was determined from the fluorescence investigation and release matrix.

Figure 2. Schematic of the hyperspectral imaging system (A) and a structure of the 3D Hypercube (B).
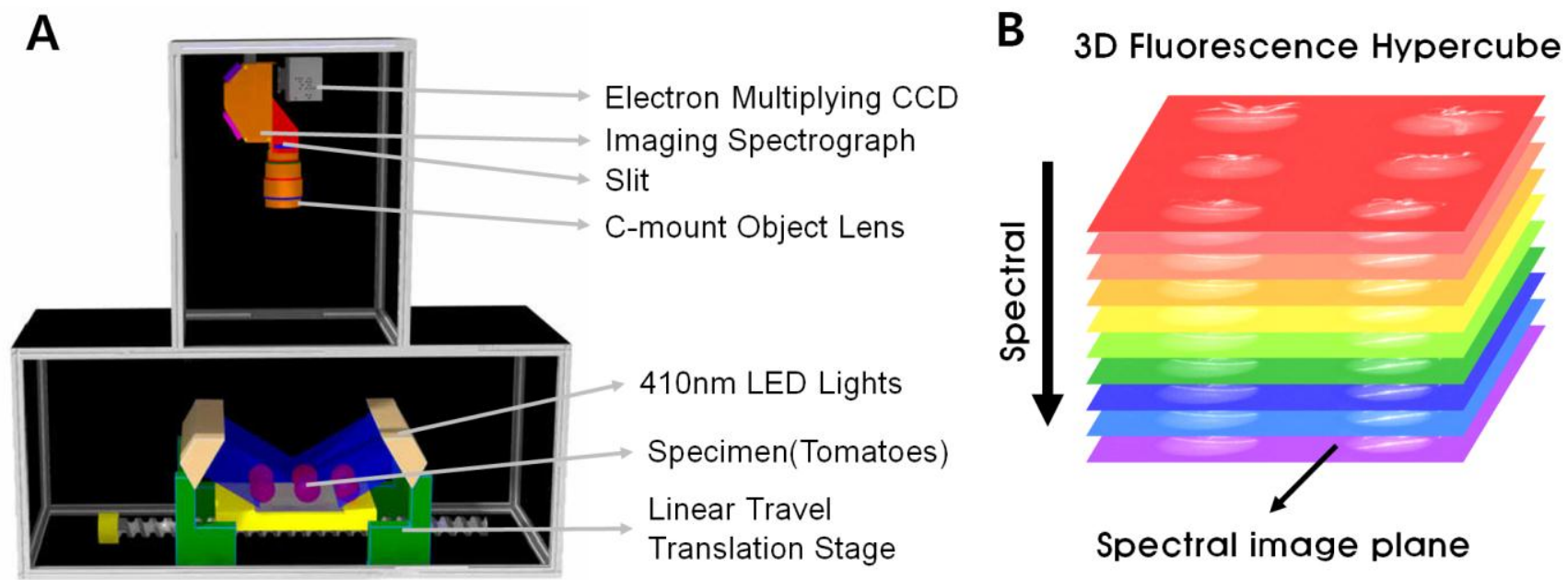

\subsection{Data Analysis}

EEM data was stored as Unsigned 64-bit integers; the 14-bit hyperspectral image was stored and analyzed as Unsigned 16-bit integers. The EEM and hyperspectral image data were processed in Matlab software (version 7.0.4, Mathworks, Natick, MA, USA). The optimum excitation waveband was selected by analyzing the EEM data by two-way ANOVA and principal component analysis (PCA). In this study, EEM data analysis yielded total emission characteristics according to the excitation of each part of the fruit (e.g., crack vs. sound, crack $v s$. stem). We then analyzed and selected an excitation waveband that could distinguish defect tomatoes by fluorescence solidity values of parts (sound surface, stem, defect area). To select the excitation waveband in the hyperspectral image system, we extracted spectral information from each part (sound surface, stem, crack area) in the hyperspectral fluorescence image. Data was analyzed by the PCA method to select the optimal emission waveband for detection of cherry tomato surface defects [10].

\section{Results and Discussion}

\subsection{Fluorescence Attributes of Stem, Sound Skin, and Cracks}

Three-dimensional (3-D) graphs for the average fluorescence emission and excitation matrix of defect tomato, stem, and normal cuticle were plotted with respect to excitation wavelength ( $\mathrm{x}$-axis), emission wavelength (y-axis), and RFI (z-axis) (Figure 3). The right contour plot in Figure 3 indicates the excitation $(350 \sim 665 \mathrm{~nm})$ and emission wavelengths $(400 \sim 700 \mathrm{~nm})$ used in this study. At excitation 250 300 nm, defect and stem parts release UV fluorescence $(300 \sim 400 \mathrm{~nm})$, similar to the reaction of general proteins [11]. However, the protein is not target component to detect crack, we did not used excitation below $350 \mathrm{~nm}$. Defect parts could be detected by fluorescence at 350 580 nm (with excitation 
$350 \sim 480 \mathrm{~nm}$ ) due to the flavonoids, carotenoids, phenolic compounds, cell walls, and anthocyanins [12]. In detail, flavonoid emission band were identifiable at 300, 450 500 and 570 580 nm with excitation at 266,355 , and $480 \mathrm{~nm}$, respectively, while anthocyanin fluorescent spectrum was peaked at 380 and 450 at 266 and 355-400 nm of excitations, respectively. The carotenoids spectrum was observed at the range from 420 to $580 \mathrm{~nm}$. Also, cell walls were responsible for fluorescence emission at 450 500 nm [13]. In addition, the spectral patterns and intensity of the compounds are different. As the objective of this study is to distinguish defect from other areas, the spectrum of these compounds do not have to be differentiated. Rather, it is important that the spectrum of them allow us to detect defect areas from stem and sound surface. Stems emitted 650 700 nm of fluorescence at excitation wavelengths ranged from $350 \mathrm{~nm}$ to $650 \mathrm{~nm}$ because of the presence of chlorophyll [14]. However, emission from intact cuticle is too weak to detect fluorescence. This might be due to waxy nature of intact cuticle as previously reported that the light and UV absorbance of waxes were drastically reduced and reached almost zero above about $350 \mathrm{~nm}[15]$.

Figure 3. Averaged EEM of each data set of (A) crack; (B) sound skin; (C) stem with the spectral rage of excitation from 200 to $655 \mathrm{~nm}$ and emission from 225 to $700 \mathrm{~nm}$.

A
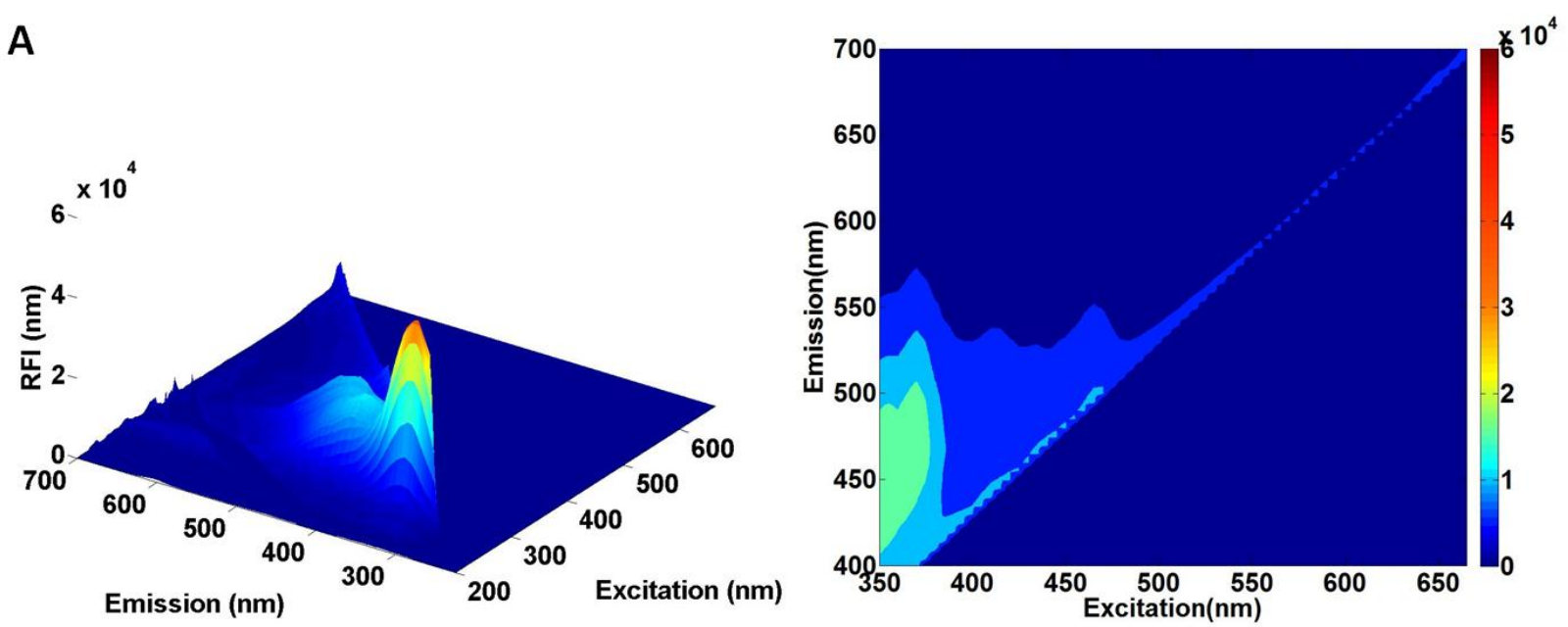

B
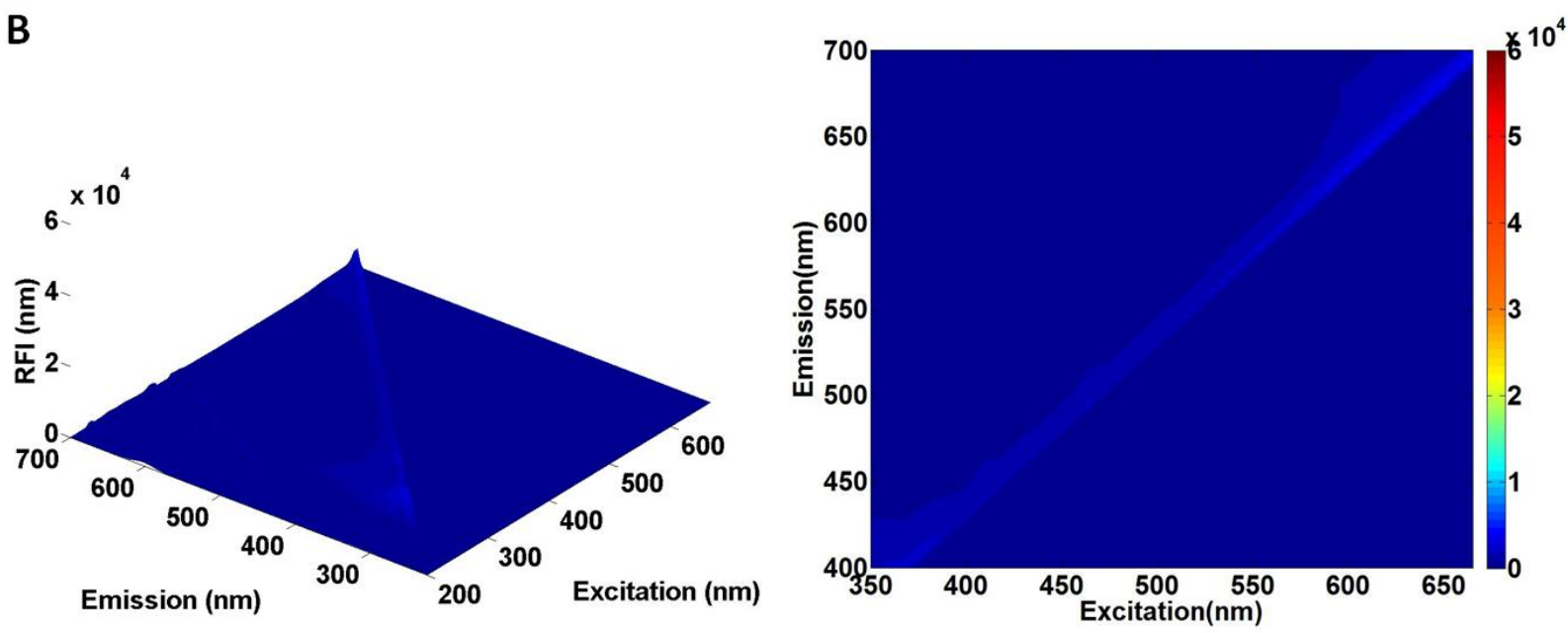
Figure 3. Cont.
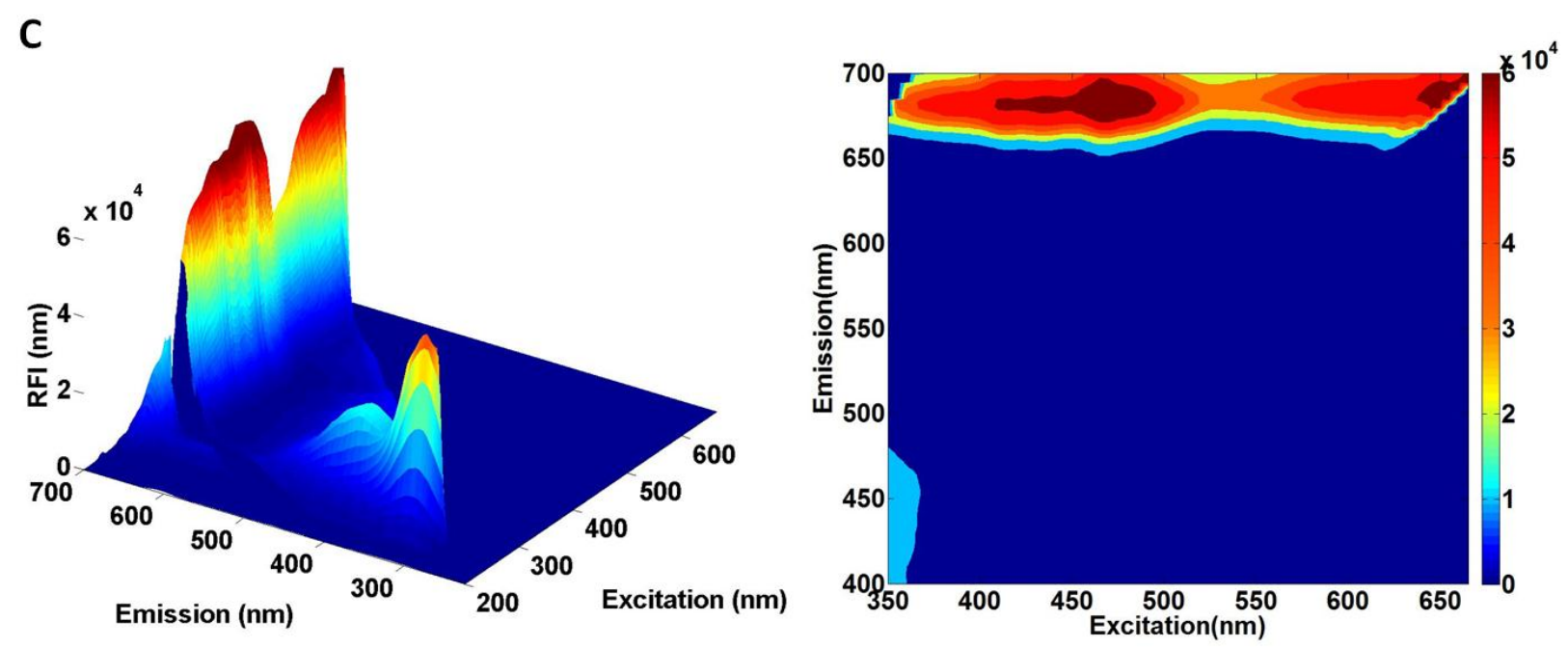

\subsection{Selection of the Excitation Waveband}

The optimal excitation and emission wavelengths have been selected to efficiently distinguish defect, stem, and normal cuticle. The emission and excitation of each part of the cherry tomato was characterized by F-value obtained from ANOVA with comparing two groups (Figure 4). A large F value indicates a more statistically significant mean separation between groups [16]. To eliminate intact parts and distinguish them from cracked parts in fluorescence images, we compared crack with stem and sound surface using ANOVA. The crack and stem were distinguishable at $480 \mathrm{~nm}$ excitation because of the presence of chlorophyll detectable by red to far-red fluorescence. Cracks and sound surfaces were clearly distinguishable at $380 \mathrm{~nm}$ excitation. This is because that fluorescence emission from cracked parts exists in the blue region while there is no fluorescence from a sound surface with the excitation wavelength of $380 \mathrm{~nm}$.

Figure 4. $F$ values of wavelength used for discriminating defect area from sound and stem on the cherry tomatoes.

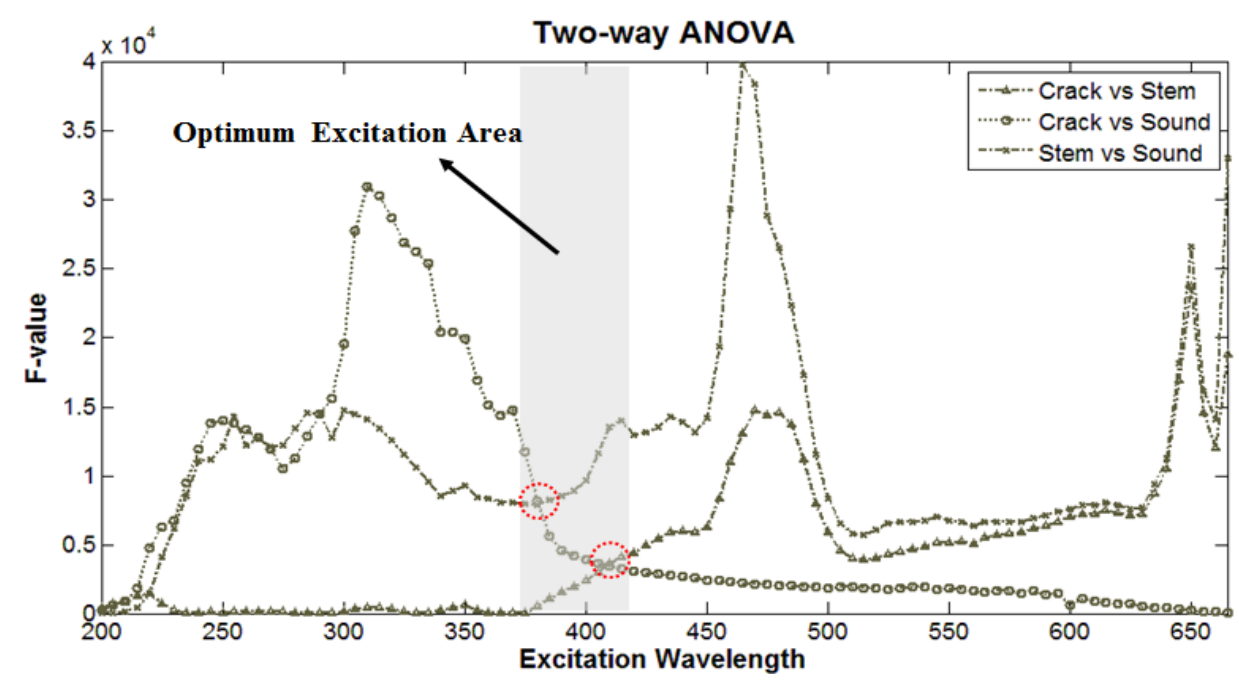


In order to find optimal excitation waveband, two-way ANOVA method between the two groups (e.g., crack area $v s$. stem) was used. Emission characteristics by excitation waveband of each part were expressed as F-values in pairwise group comparisons (Figure 4). The highest F-value for discriminating defect areas from stem areas was $320 \mathrm{~nm}$, and the F-value for distinguishing stem from sound surface and crack was peaked at $460 \mathrm{~nm}$. Consequently, the optimal excitation wavebands for differentiating 3 areas of tomatoes (defect, stem and sound areas) were 380 410 nm determined at the intersection points of two plots in Figure 4.

\subsection{Analysis of Fluorescence Images}

Figure 5 illustrates the average fluorescence spectrum of each part (defect area, sound surface, and stem) extracted from hyperspectral fluorescence images obtained with $410 \mathrm{~nm}$ excitation wavelength. In the blue-green spectrum $(480 \sim 550 \mathrm{~nm})$, defect tomato parts yielded strong fluorescence, while stem emitted strong fluorescence in the red region $(680 \sim 700 \mathrm{~nm}$ ) (Figure 5). However, fluorescence of the intact fruit was consistently low. The results showed similar patterns with the results derived from EEM analysis.

Figure 5. Mean fluorescence emission spectra of sound surface, defect area, stem area in hyperspectral image of cherry tomatoes upon excitation by $410 \mathrm{~nm}$ light source.

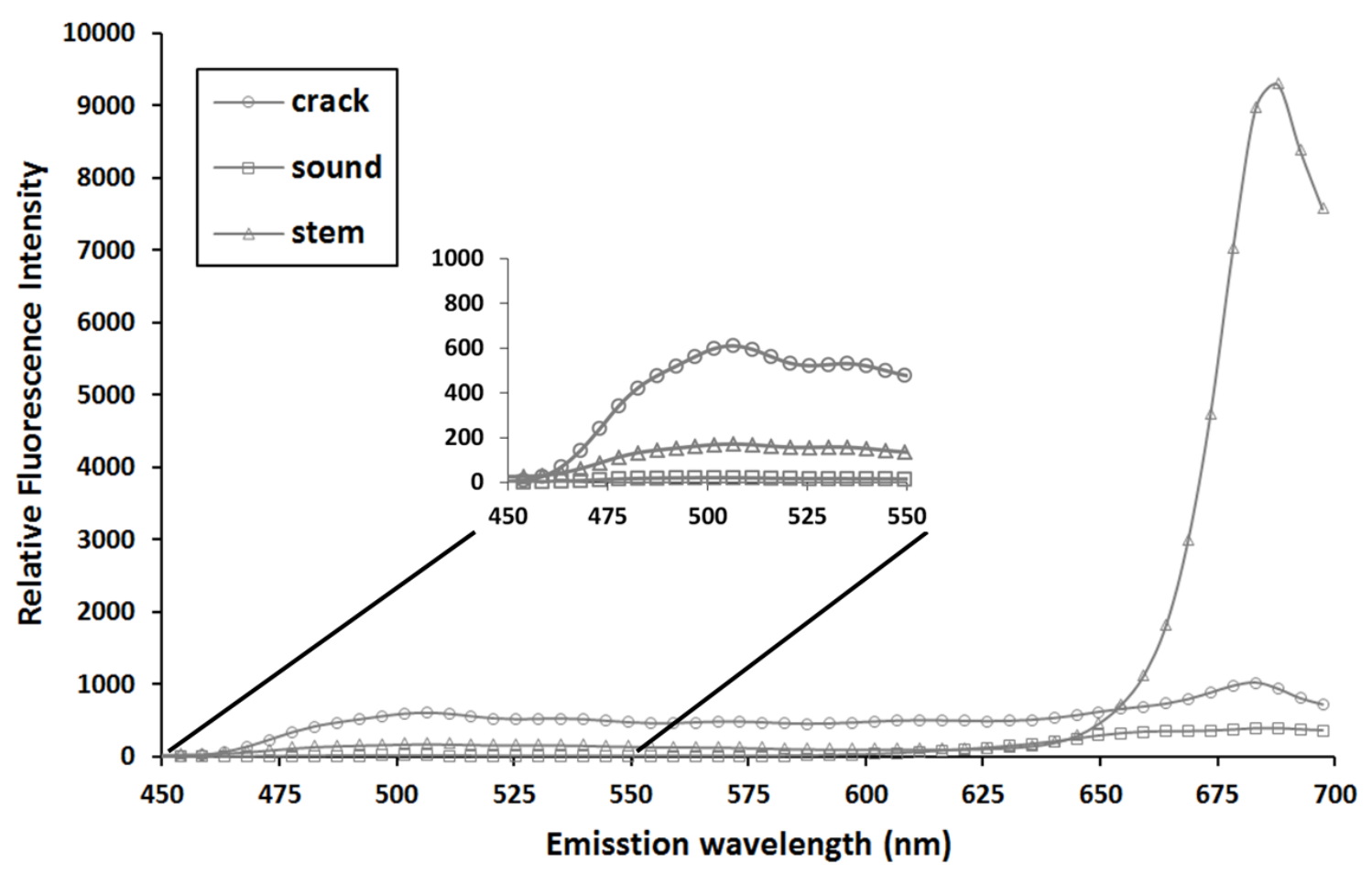

To detect defect tomatoes using the emission spectrum at $410 \mathrm{~nm}$ excitation, we selected the significant multi-waveband analyzed by PCA. Figure 6 shows the weighting coefficients for PCA (PC1-PC4). The peaks and valleys signify the dominant waveband for each PC which transforms the original wavelengths by a linear sum at each wavelength multiplied by calculated weighting coefficients. The maxima for each PC were observed at $688 \mathrm{~nm}$ for PC1, $506 \mathrm{~nm}$ for PC2, $674 \mathrm{~nm}$ for PC3, and $700 \mathrm{~nm}$ for PC4. Fluorescence release was the greatest from stem with the maximum weighting 
coefficient of PC1 at $688 \mathrm{~nm}$, and the weighting coefficient of PC2 appeared at $506 \mathrm{~nm}$, the fluorescence wavelength of cracked tissue. These results were consistent with the EEM analysis.

Figure 6. Spectral weighting coefficients for principal components.

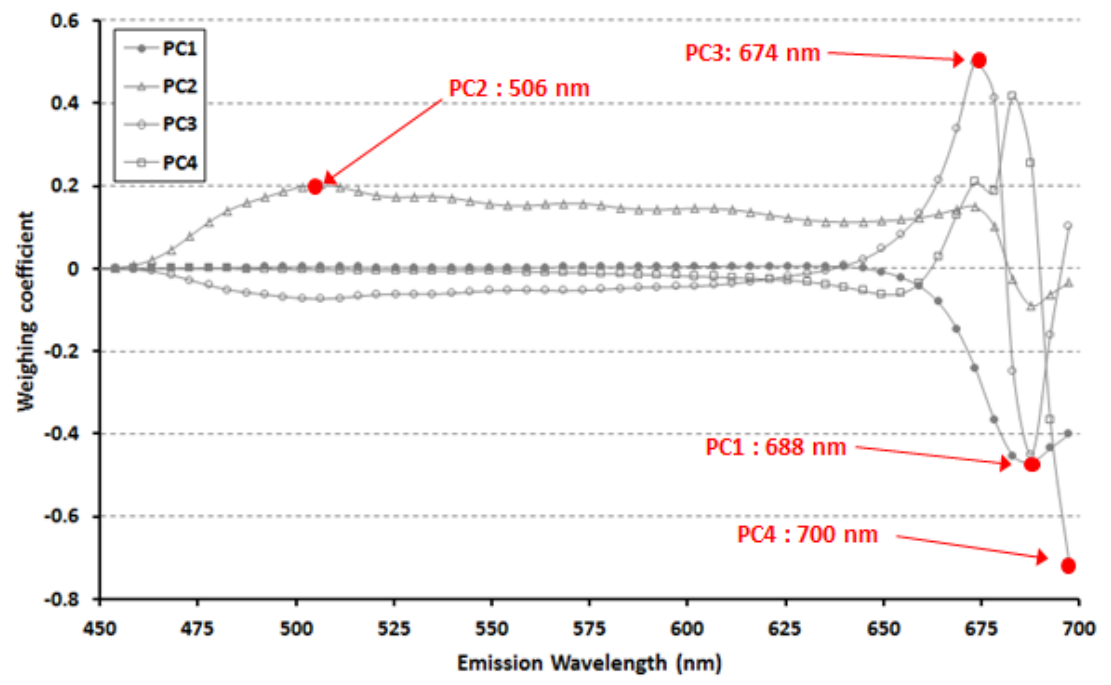

\subsection{Detection of Defect Cherry Tomatoes}

Detection of damaged areas is difficult with a single spectral band. Hence, PCA was performed for the entire spectral data of defect, stem and sound surface. A principal component (PC) image was generated from the data at $688 \mathrm{~nm}$ and $506 \mathrm{~nm}$ (Figure 7). As aforementioned, PC is a new variable created by linear combination of original variables, i.e., emission wavelengths. Different numbers in PC means they are generated by different coefficient in linear combination. Generally, higher PC can explain more variations in data. The total image for PC1 yielded a precise image of the stem, and defects and stems appeared in the PC2 image. To detect defect tomatoes, the PC2 image after erasing stem part using the PC1 image was used and a histogram was produced by extracting the PC1 and PC2 values for stems, cracks, and sound surface (Figure 8). The PC1 value of cracks was distributed across 50-1700, while the PC1 value of stems was concentrated around 15,800. In the PC2 image, the value for cracks was distributed from -1900 to -100 , and the value for sound surfaces peaks from -50 to -20 .

Figure 7. PC images by using two wavebands.

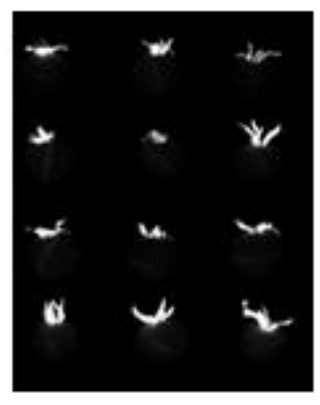

PC1

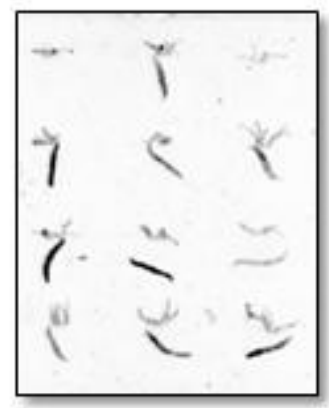

PC2 
Figure 8. PC value about each part by histogram: (a) crack (PC1); (b) stem (PC1); (c) crack (PC2); and (d) sound area (PC2). The x-axis indicates PC value and the y-axis indicates frequency.

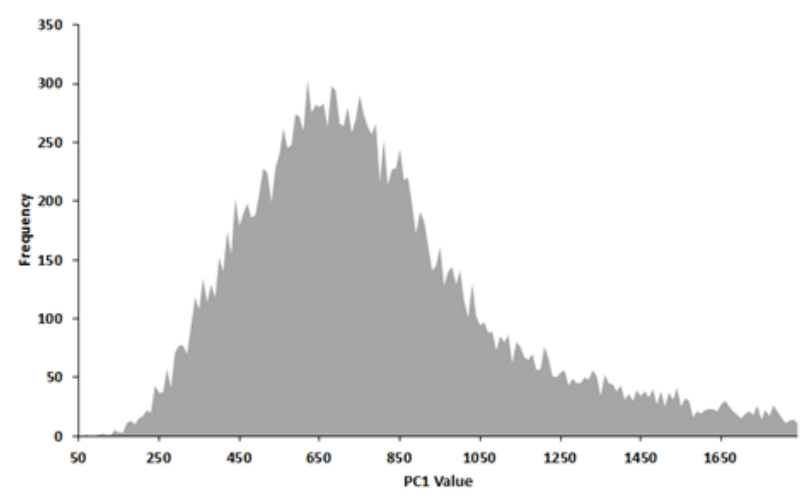

(a)

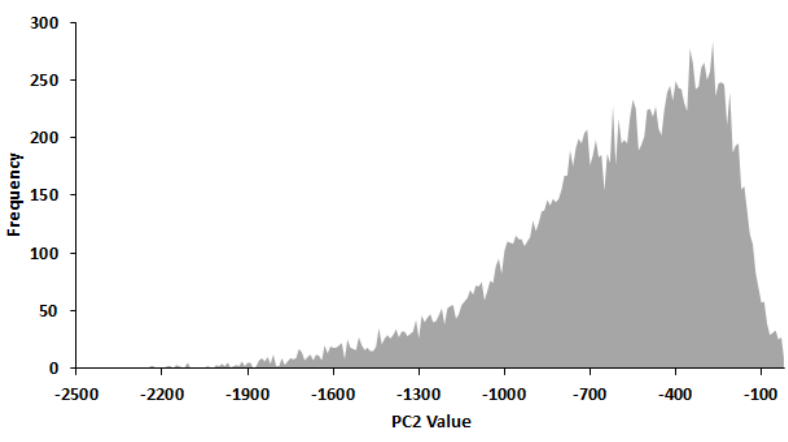

(c)

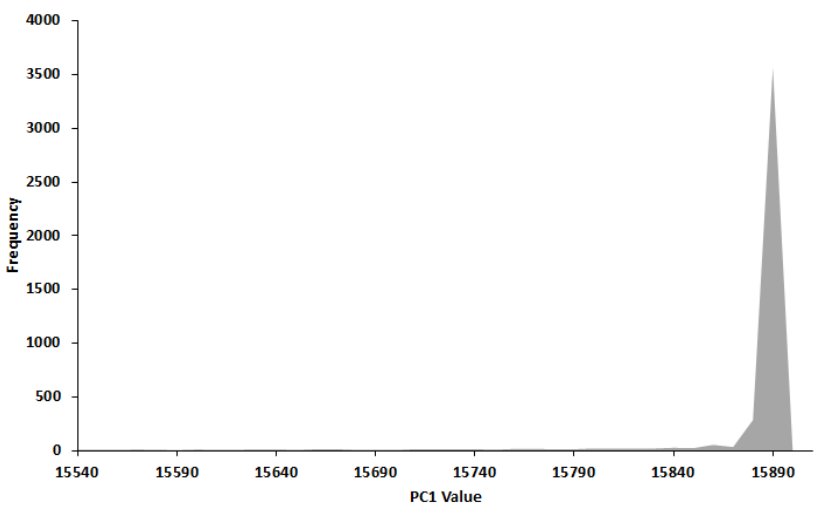

(b)

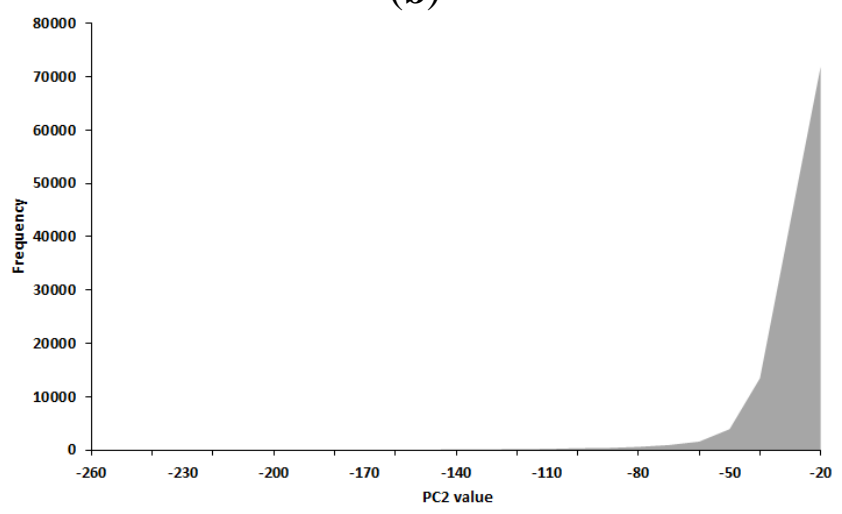

(d)

To generate a binary image by applying threshold value of each PC image, it was necessary to establish a global threshold value that could distinguish the two groups. We expressed the distinction accuracy with the applied threshold in Figure 9; the optimal thresholds were 2175 and -113 (points of intersection) for discriminating stem from crack areas and sound surfaces from crack areas, respectively.

Figure 9. Classification aaccuracy when threshold value of two groups is applied: (a) stem vs. crack; (b) sound area vs. crack.

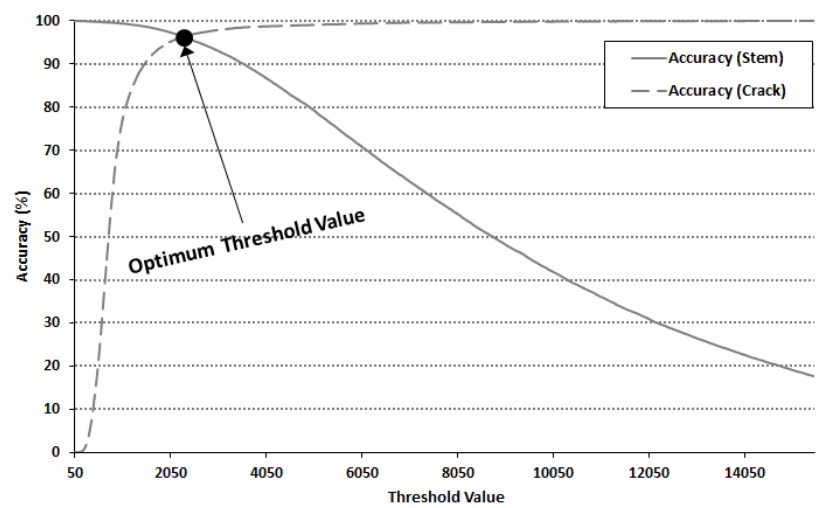

(a)

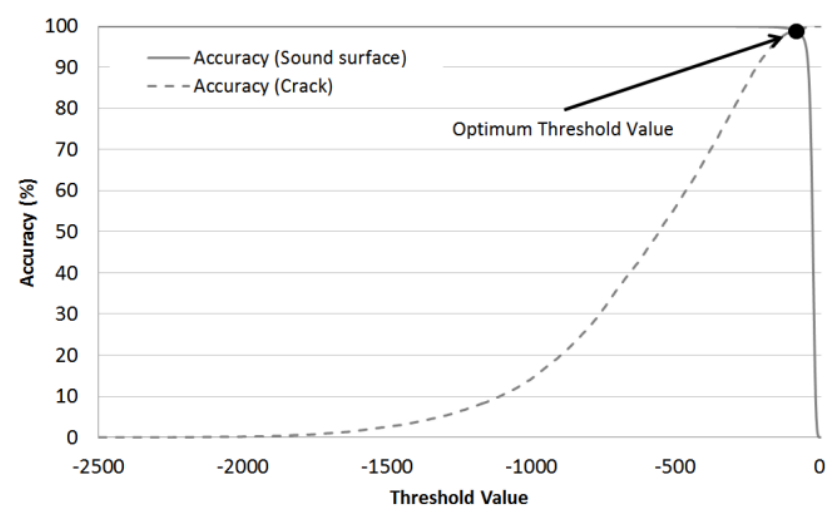

(b) 
Defect areas on the cherry tomatoes were detected by using multispectral image processing methods (Figure 10). The experimentally derived fluorescence images at 506 and $688 \mathrm{~nm}$ were used to generate PC images so that the stem and the crack areas were emphasized. The PC1 and PC2 binary images were generated by applying the global threshold values to detect the stem and the crack areas only. The PC1 binary image was used to erase the stem parts from the PC2 image so that only the crack areas could be detected. In addition, waveband images of $700 \mathrm{~nm}$ were subjected to a smoothing method, followed by thresholding at a specific threshold value to discriminate samples from background. The threshold image was used to apply a mask for the resultant PC2 binary image. The masked PC2 binary image was the resultant image displaying only the defect areas on the cherry tomatoes.

Figure 10. Image processing for detecting defects of cherry tomatoes.

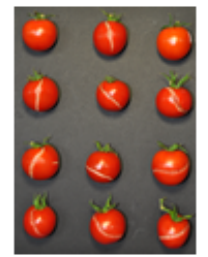

Original image
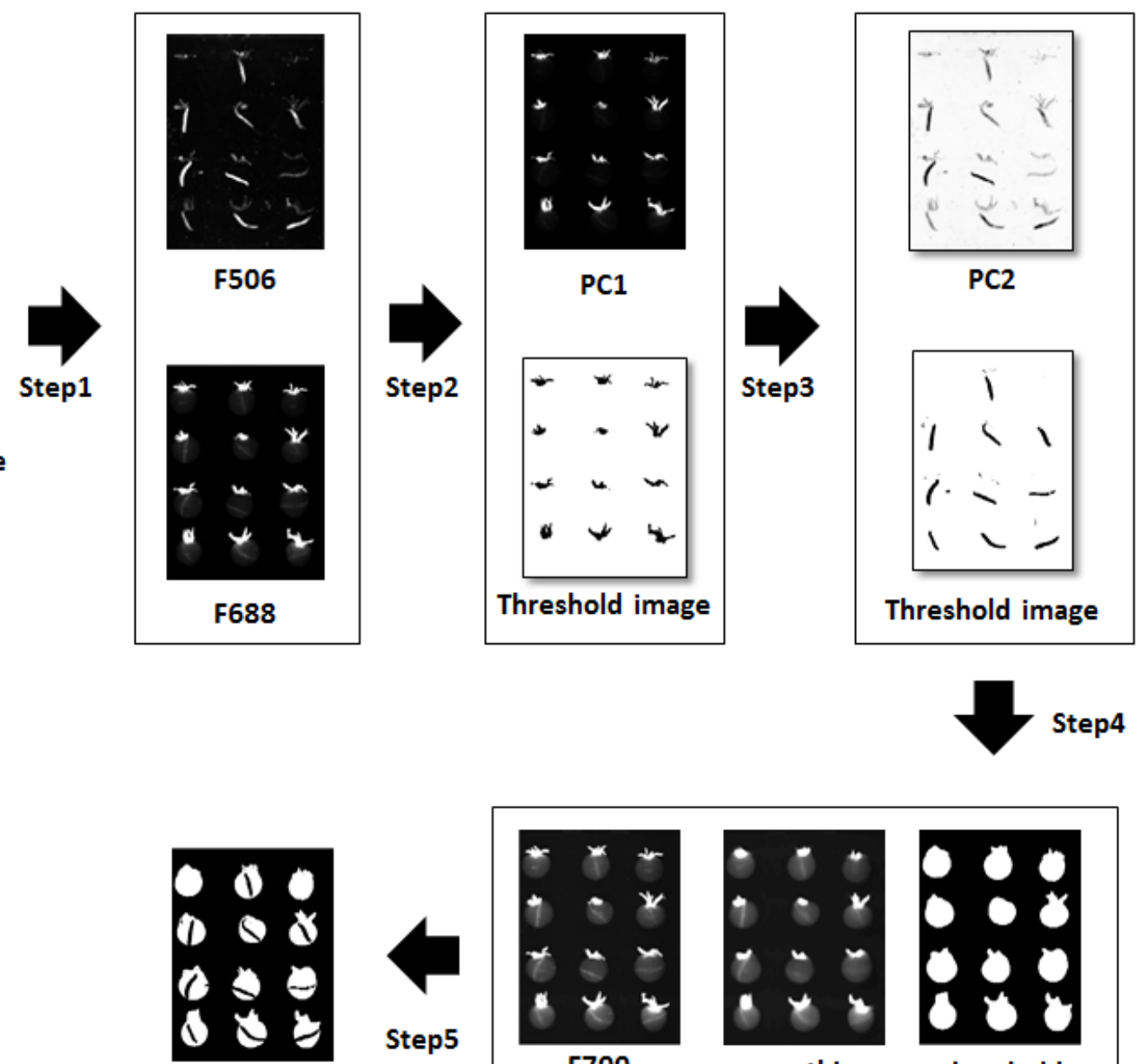

Final image
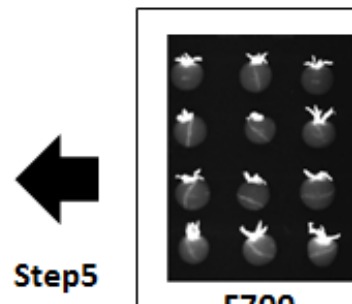

F700

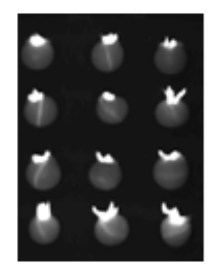

smoothing

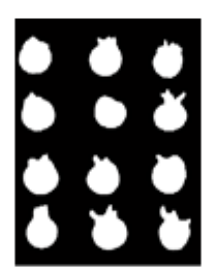

threshold

The actual sample images and detection results are displayed in Figure 11. The green circle in the binary image indicates a normal cherry tomato with no defects. In contrast, red circles indicate defect tomatoes. With this process, 121 defect and 23 normal tomatoes were imaged, resulting that defect tomatoes could be detected with $>98 \%$ accuracy (Table 1). Three failures might be due to subtle fluorescence differences between defects and sound surface, resulting in the detection error. To improve the accuracy, even though it's still enough high, the hyperspectral fluorescence imaging system or statistical algorithm might be adjusted. 
Figure 11. Resultant detection images.

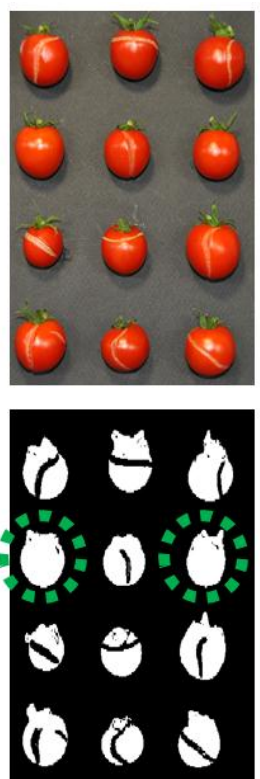

Sample 1

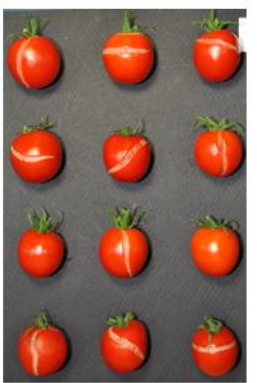

$0 \Leftrightarrow \theta$

$\theta$ \& 8

0.0. $\Delta \Delta \theta$

Sample 7
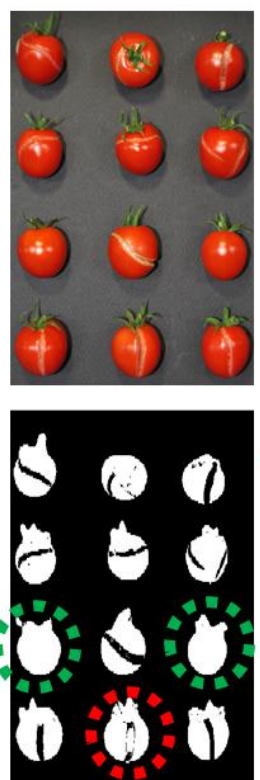

Sample 2

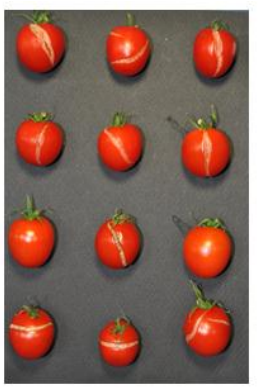

000
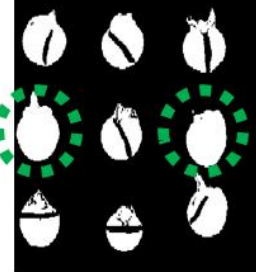

Sample 8
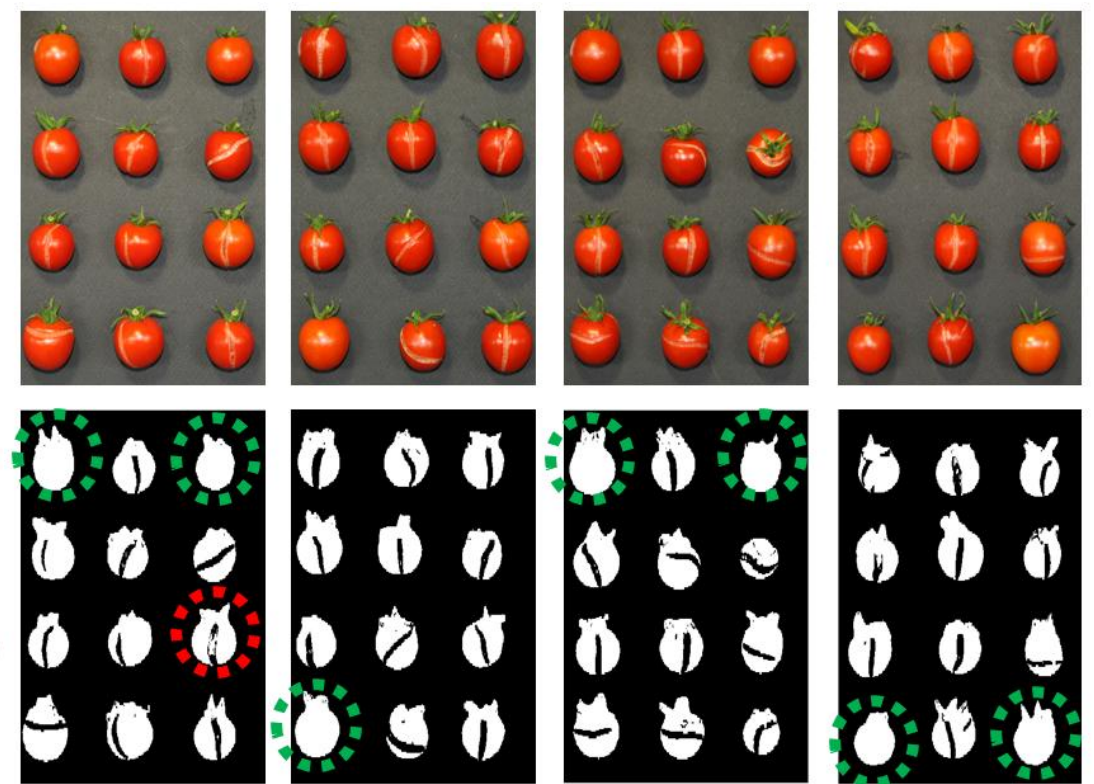

Sample 3
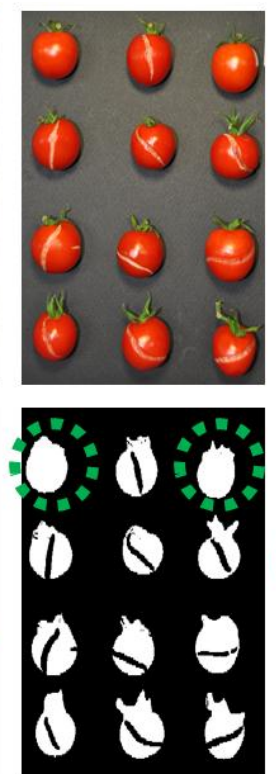

Sample 9

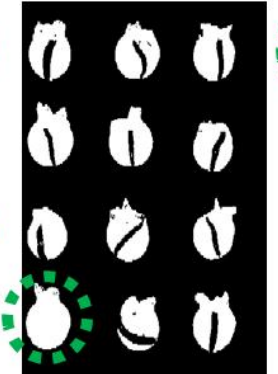

Sample 4
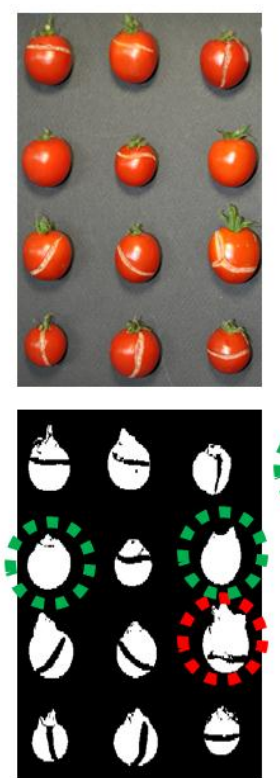

Sample 10

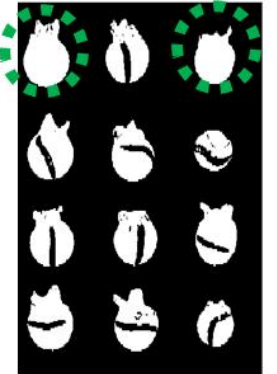

Sample 5
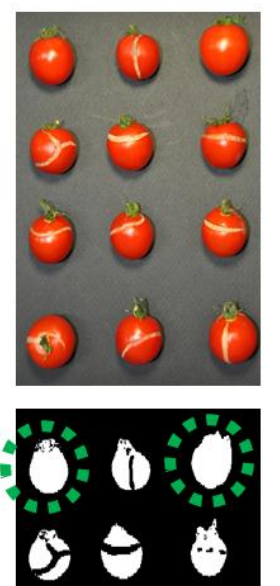

$\theta 4 \theta$

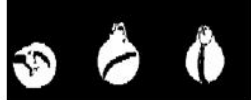

Sample 11

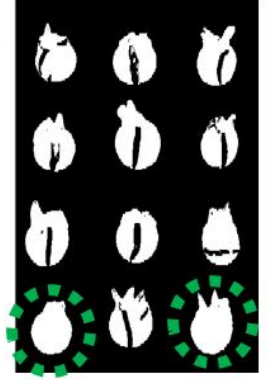

Sample 6
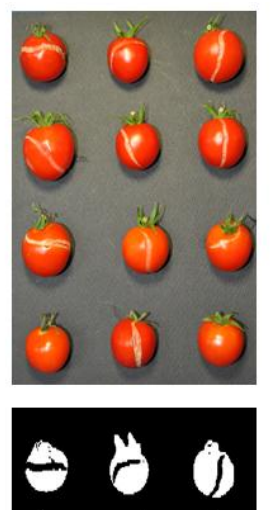

000
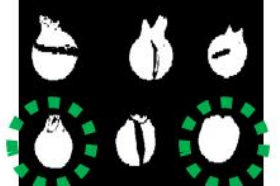

Sample 12

Table 1. Classification results.

\begin{tabular}{ccc}
\hline Intact Cherry Tomatoes & Defect Cherry Tomatoes & Accuracy (\%) \\
\hline $23 / 23$ & $118 / 121$ & 98 \\
\hline
\end{tabular}

\section{Conclusions}

The goal of this study was to develop a simple image processing method based on multispectral fluorescence imaging for onsite detection of cuticle defects in cherry tomatoes. We found that cherry tomato features yield significant fluorescence emission wavebands at specific excitation wavelengths. These attributes enable discrimination of defect from intact cherry tomato surfaces. Excitation at $410 \mathrm{~nm}$ was used to distinguish defects from the sound surfaces and stems. To detect defects on cherry 
tomatoes by using multispectral fluorescence images, the optimal wavebands were determined by PCA to be 688 and $506 \mathrm{~nm}$. Dual-fluorescence imaging at 688 and $506 \mathrm{~nm}$ provided defect detection with $98 \%$ accuracy. We have demonstrated the feasibility of using hyper- and multispectral imaging to detect defect cherry tomatoes. The technology can be applied for quality control in cherry tomato production.

\section{Acknowledgments}

This research was supported by Technology Commercialization Support Program, Ministry of Agriculture, Food and Rural Affairs (MAFRA), Republic of Korea.

\section{Author Contributions}

In-Suck Baek and Byoung-Kwan Cho designed the experiments and wrote the manuscript. Moon S. Kim and Hoonsoo Lee performed the experiments. Wang-Hee Lee helped to analyze the data and modify the article.

\section{Conflicts of Interest}

The authors declare no conflict of interest.

\section{References}

1. Vargas, A.M.; Kim, M.S.; Tao, Y.; Lefcourt, A.M.; Chen, Y.R.; Luo, Y.; Buchanan, R. Detection of fecal contamination on cantaloupes using hyperspectral fluorescence imagery. J. Food Sci. 2005, 70, e471-e476.

2. Jeong, D.; Kim, M.S; Lee, H.; Lee, H.; Cho, B. Detection algorithm for cracks on the surface of tomatoes using multispectral Vis/NIR reflectance imagery. J. Biosyst. Eng. 2013, 38, 199-207.

3. Cho, B.; Kim, M.S.; Baek, I-S.; Kim, D-Y.; Lee, W-H.; Kim, J.; Bae, H.; Kim, Y-S. Detection of cuticle defects on cherry tomatoes by using hyperspectral fluorescence imagery. Postharvest Biol. Technol. 2013, 76, 40-49.

4. Kim, M.S.; Chen; Y.R.; Cho, B.; Chao, K.; Yang, C.C.; Lefcourt, A.M.; Chan, D. Hyperspectral reflectance and fluorescence line-scan imaging for online defect and fecal contamination inspection of apples. Sens. Instrum. Food Qual. Saf. 2007, 1, 151-159.

5. Cho, B.; Kim, M.S.; Chao, K.; Lawrence, K.; Park, B.; Kim, K. Detection of Fecal Residue on Poultry Carcasses by Laser Induced Fluorescence Imaging. J. Food Sci. 2009, 74, E154-E159.

6. Kim, T.; Lee, H.; Kim, M.S.; Lee, W.H.; Cho, B. Optimal optical filters of fluorescence excitation and emission for poultry fecal detection. J. Biosyst. Eng. 2012, 37, 265-270.

7. Ariana, D.P.; Lu, R. Hyperspectral waveband selection for internal defect detection of pickling cucumbers and whole pickles. Comput. Electron. Agric. 2010, 74, 137-144.

8. Qin, J.; Chao, K.; Kim, M.S.; Kang, S.; Jun, W. Detection of organic residues on poultry processing equipment surfaces by LED-induced fluorescence imaging. Appl. Eng. Agric. 2011, 27, 153-161.

9. Kim, T.M.; Cho, B.; Kim, M.S. Emissions filter design to detect poultry skin tumors using fluorescence hyperspctral imaging. Rev. Colomb. Cienc. Pecu. 2010, 23, 9-16. 
10. Baek, I.S.; Cho, B.; Kim, M.S.; Kim, Y.S. Determination of optimal excitation and emission wavebands for detection of defect cherry tomato by using fluorescence emission and excitation matrix. Proc. SPIE 2011, doi:10.1117/12.2018543.

11. Corp, L.A.; McMurtrey, J.E.; Chappelle, E.W.; Daughtry, C.S.; Kim, M.S. UV band fluorescence (in vivo) and its implications for the remote assessment of nitrogen supply in vegetation. Remote Sens. Environ. 1997, 61, 110-117.

12. Lia, A.; Santangelo, E.; Soressi, G.P.; Fantoni, R. Analysis of the main secondary metabolites produced in tomato epicarp tissue during fruit ripening using fluorescence techniques. Postharvest Biol. Technol. 2007, 43, 335-342.

13. Harris, P.J.; Hartley, R.D. Detection of bound ferulic acid in cell walls of the graminae by ultraviolet fluorescence microscopy. Nature 1976, 259, 508-510.

14. Kim, M.S.; Lefcourt, A.M.; Chen, Y.R. Multispectral laser-induced fluorescence imaging system for large biological samples. Appl. Opt. 2003, 42, 3927-3934.

15. Jacobs, J.F.; Koper, G.J.M.; Ursem, W.N.J. UV protective coatings: A botanical approach. Prog. Org. Coat. 2007, 58, 166-171.

16. Cho, B.; Chen, Y.; Kim, M. Multispectral detection of organic residues on poultry processing plant equipment based on hyperspectral reflectance imaging technique. Comput. Electron. Agric. 2007, 57, 177-189.

(C) 2014 by the authors; licensee MDPI, Basel, Switzerland. This article is an open access article distributed under the terms and conditions of the Creative Commons Attribution license (http://creativecommons.org/licenses/by/4.0/). 